\title{
B-Cell CLL/Lymphoma 7 Protein Family Member C
}

National Cancer Institute

\section{Source}

National Cancer Institute. B-Cell CLL/Lymphoma 7 Protein Family Member C. NCI

Thesaurus. Code C157261.

B-cell CLL/lymphoma 7 protein family member C (217 aa, $\sim 23 \mathrm{kDa}$ ) is encoded by the human BCL7C gene. This protein may be involved in nucleosome remodeling and apoptosis. 